\title{
Survey on Different Smart Parking Techniques
}

\author{
Minla Chandrahasan \\ University of Mumbai \\ 4, Sarovar CHS, Sec-9A \\ Vashi, Navi Mumbai, India
}

\author{
Ankita Mahadik \\ University of Mumbai \\ 74/2191, Pantnagar, \\ Ghatkopar(E), Mumbai, India
}

\author{
Tejal Lotlikar \\ University of Mumbai \\ B-301, New Fulora CHS \\ Dombivali, Mumbai, India
}

\author{
Madhusmita Oke \\ University of Mumbai \\ 15-201, Regency Estate \\ Dombivali, Mumbai, India
}

\author{
Anjali Yeole \\ University of Mumbai \\ 1009-C, Kukreja Residency \\ Chembur, Mumbai, India
}

\begin{abstract}
The increase in city traffic is one of the major effects of population growth especially in urban areas. Due to this searching for a vacant parking area has become difficult during peak hours. In this paper, we have conducted a survey on different available smart parking techniques, their drawbacks and proposed solutions. As smart parking has become the need of the day, in this paper we present different innovative techniques which exploit latest technology to provide better services to the end users as well as reduce the workload of the parking administrator. Sections II to V describe existing systems and sections VI to VIII describe suggestions for a cloud application.
\end{abstract}

\section{Keywords}

Smart Parking, ParkNet, Crowd sourcing, Cloud.

\section{INTRODUCTION}

Searching for a parking lot possibly closest to the intended destination has become a cumbersome task due to the increasing vehicular traffic in the urban areas. It leads to wastage of time and fuel as well as creates a negative impact on the environment. The drivers keep searching for suitable parking lot which leads to increase in traffic and aggravation of the drivers.

In order to resolve these issues and satisfy the increasing demand for the parking areas, parking management organizations are trying to implement better and technologically advanced solutions. A variety of methodologies have beenimplemented to disseminate parking availability information through various platforms.

The real time parking availability monitoring enables advance parking spot reservation. This improves the level of services provided to the customers and also improves the overall performance of the system.

In this paper, we present the latest technologies used in smart parking which have been proposed to resolve the issues encountered in existing systems. The various methodologies discussed in this survey include parking availability monitoring and parking lot reservation using techniques like RFID, Crowdsourcing along with various sensors.

\section{SMART PARKING APPLICATIONS USING RFID TECHNOLOGY [1]}

RFID is an unmanned, secure, automation technology with main components as RFID readers, labels, computers, barriers, software etc. The software is for controlling operations, reporting transactions and managing parking lot information.

\subsection{Advantages of RFID technology}

1. Manually achieved workloads are decreased considerably.

2. Efficient in inventory tracking technology.

3. Easy installation and operation, automated data gathering and reporting.

\subsection{Features of the system}

1. Avoids traffic jam as vehicles are not stopped at the entrance.

2. No payment collected after every check-out. Monthly fees will be calculated and drawn from the bank accounts issuing an invoice for each transaction.

\subsection{Design}

1. Timing of the gates and additional sensors prevent multiple check-in and check-out. RFID readers control check-in and check-out barriers.

2. 40-bit identification data on RFID label is read from approximately $7.62 \mathrm{~cm}$ distance using RFID reader.

3. Centralized management of Database to enable remote access and administration of the system over the internet.

4. Database consists of main 2 tables:

VehicleInformation (General information about the vehicle like vehicleID, plateNo, type, model)

VehicleCirculationInfo (check-in and check-out attempts dates, time, total fees)

5. RFID reader is connected to computer's USB port by USB cable for communication between developed software and RFID reader. Also, there is another USB connection for barrier as well. One barrier and one RFID reader are used for each parking lot.

6. When a registered vehicle arrives at the check-in point of a parking lot, the system verifies if it is registered or not. If it is so, and no previous check-in or check-out records exist for it, the check-in information gets stored in the database and the barrier will lift for the vehicle to proceed.

7. At the check-out, a vehicle's identification information is verified with the database first. If the vehicle is a registered one and its access is authorized, the system permits its check-out. During check-out, the system 
locates the vehicle's check-in details and updates it with the check-out details.

8. LCD displays directly connected to the RFID reader can be located at parking-lot entrances in order to conveniently inform drivers about the availability of free parking space. Thus time won't be wasted looking for park space.

9. A vehicle that checks-in to a parking-lot without an RFID notification, shall be restricted from checking-out later, thereby avoiding unauthorized entries.

\subsection{Drawbacks}

1. Expensive, tags are application specific, no one tag fits all, more than one tag can respond at the same time, internet disconnection.

2. If two vehicles will enter the parking-lot side by side, within range of the RFID reader, the system will not read either of their identification information and process it.

3. Electromagnetic spectrum signals are relatively easy to jam using energy at the right frequency.

\subsection{Improvements}

1. Cost effective technology like OCR should be used.

2. Use a local server too, which will run when the connection to central database is lost.

3. Parking-lot entrance design must enable passage of only one vehicle at a time.

\section{RESERVATION BASED SMART PARKING SYSTEM [2]}

\subsection{Goals and objectives:}

Reservation based smart parking system aims at broadcasting real time parking information to drivers via an application. It will provide service for reservation of parking space in advance. This will enable drivers to retrieve parking information in specific parking district. Unlike traditional manual parking system, smart parking will enhance the service at large. It will beequipped with payment option including dynamic pricing for users.

\subsection{Advantages}

1. Reduction in searching time for parking lot.

2. Remarkable reduction in traffic congestion.

3. The above factors will in turn reduce energy consumption and pollution.

\subsection{Performance Metrics and Challenges}

Reservation based parking system enables searching most convenient parking space which is time efficient. It avoids bottleneck when drivers try to book specific lot simultaneously that is done by means of time stamp synchronization. The system collects and stores data namely status of parking space, reservation time, location, driver's identity. This is stored in repository and mirror database (for convenience of access). The system verifies driver's identity before allowing access to the privileges.

\subsection{Architecture and Design}

The sensor system in the parking lot updates availability status of the lot. The information is collected and updated by central system that will reflect changes in the application used by the user. As a result, the users can reserve space accordingly. The system dynamically decides price of booking on the basis of traffic, availability, peak hours etc (see figure 1)

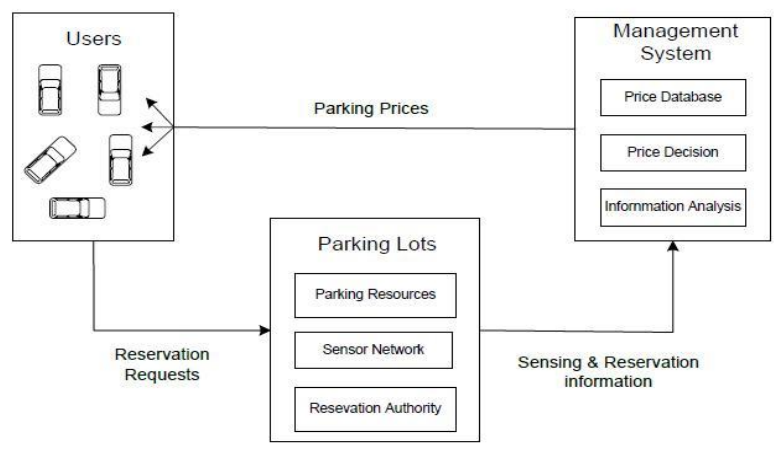

Figure 1: System architecture

\subsection{Hardware}

The system mainly uses Zigbee sensors and smart phone support for deployment. Light and vibration sensors detect vehicles present in the lot. Bluetooth module is responsible for communication between user and the sensor information. The identity of the user is also detected by means of Bluetooth

\subsection{Software}

The user end is provided with an application that serves as an interface for reservation. The basic functions that are undertaken by the application software present are:

1. Reservation data analysis

2. Dynamic price decider

3. Payment option

4. Retrieval of data

5. Synchronization of data between mirror database and repository

6. Authentication of user

7. Web service for obtaining real time parking information

\subsection{Drawbacks}

1. The deployment of this system makes use of Zigbee sensors and Bluetooth enabled modules for each parking space of car. This naturally increases the cost of the system and maintenance problems.

2. The communication of Bluetooth module is less robust due to short range which is not acceptable in many circumstances.

3. The authentication mechanism involved is feeble due to the simple sensors involved in the process.

4. The problem of bottleneck in case of heavy traffic situations is likely

\section{SMART PARKING RESERVATION SYSTEM USING SMS [3]}

4.1 System Overview

This paper proposes a system permitting users to reserve parking space via an SMS. Once a reservation is confirmed, the users will be supplied the allotted lot number and a password to enter the parking area. If users exceed the set time to reach the reserved parking lot, the password shall expire and they will be notified using an SMS. The system is subdivided into Reservation and Access system. The reservation is handled by a micro-RTU (Remote Terminal 
Unit) configured with SSE OPC (OLE for Process Control) Server. The access system is handled by a microcontrollerPIC16F877-which measures time periods for validity of passwords, stores/provides information on the availability of parking spaces as well as allowing or denying access to the parking area. A weight sensor is positioned at the lot area to sense the presence of the vehicle.

\subsection{Drawbacks}

1. Standalone system - The system is designed for individual use by parking areas, and not a centralized system encompassing reservation for all parking areas within a region

2. Scalability - As the system uses CMOS flash memory for storage of parking information, the database can be scaled within hardware limitations.

\subsection{Improvements}

1. A centralized system for all parking areas so that users can browse alternatives before deciding on making a reservation at a particular location.

2. Deploying the system on the cloud for scalable memory and implementation of appropriate queuing model to handle simultaneous reservations.

\section{PARKNET : DRIVE BY SENSING ROADSIDE PARKING STATISTICS[6]}

\subsection{Overview}

ParkNet is a mobile system comprising of vehicles that collect parking space occupancy information by driving by ParkNet vehicle is equipped with GPS receiver and passenger-side facing ultrasonic rangefinder to determine vacant parking spaces. The data collected is integrated at the central server producing a real-time map of parking availability. This is used to inform user's requests of reserving parking spaces. In order to achieve accuracy of location, environmental fingerprinting approach is devised.

\subsection{Goals and Objectives}

1. Deploying a mobile sensing approach to road-side parking availability detection by surveys of implementation over a small area

2. Improvement in the technique of GPS positioning using environmental fingerprinting necessary for accurate matching of cars and parking space.

3. Analyze the real-time map of parking occupancy generated by the system to study changes in traffic.

\subsection{Design metrics and challenges}

1. The drive-by monitoring system approach needs accurate information on hourly basis in order to map it to the image and convey occupancy to drivers and municipal government parking management stations.

2. The system must be capable of identifying appropriate user and vehicle parked for specific lot. In addition, pricing policy needs to be clear according to the map generated at particular time.

3. The system must harness the sensors that are already in use for automobiles instead of resorting to expensive sensors.

4. The feasibility of deployment must be kept in check according to the increasing number of vehicles in that particular area.

\subsection{Prototype Deployment}

ParkNet makes use of ultrasonic sensors in addition to GPS module for end to end communication. The vehicle is fitted with webcam for avoiding shift errors. The GPS module present in the automobile itself may be rewired to transfer relevant information of parking occupancy. The sensors help to identify the measure of change in distance for a particular space and nearby occupancies. This mechanism gives vacant space, if any, in the parking area. The information can be relayed to central stations using GPS.

The errors in location finding be kept in check using environmental fingerprinting. The accuracy in real time map of parking information is facilitated by GPS trip boxes. The timestamp associated with the information is checked. If it falls within the trip limits of box, it is utilized for real time map otherwise used in the next turn. This minimizes the conflicts in the image and enhances efficiency.

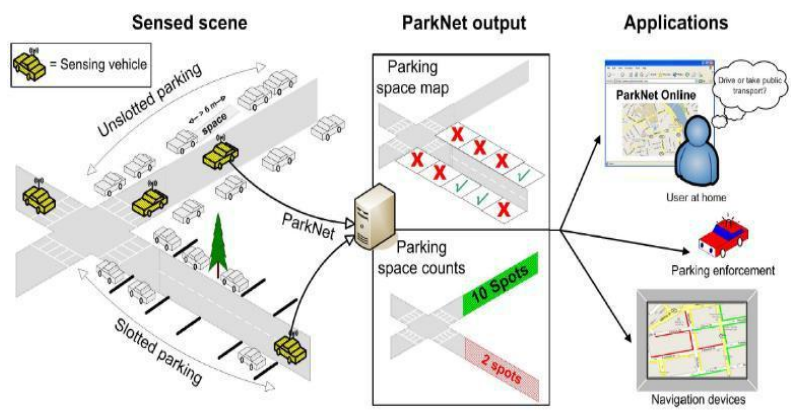

Figure 2 : Various scenarios and events involved in the detection of parking space

\subsection{Drawbacks}

3. The ultrasonic sensors may fail to detect i. distances if the speed limitations are not followed.

4. Multi-lane parking will need lane detection mechanism in addition to other detection algorithms. This renders the system even more complex.

5. The accuracy of locations and occupancy may be erroneous in case of unregistered spaces used to park or used as storage.

6. The system would need considerable investment for converting many taxicabs into ParkNet equipped cars which may not be feasible on a large scale.

\section{SMART PARKING SOLUTIONS FOR URBAN AREAS [6]}

A brief description of current status from a technological perspective is provided and results of a driver survey about smart parking facilities are provided.

There are two types of parking areas as described in given paper:

7. On street parking

8. Off street parking i.e. parking grounds or areas.

It is observed that there are more number of off street parking space available and time restrictions are less strict as compared to on street parking.

Traditional system followed is using barriers in the car parking areas. The payment takes place at entry and a record of vehicles is kept at entry and exit points to find available parking areas. Barriers rely on Automatic Vehicle Identification (AVI) system using RFID which identifies and 
detects car or vehicles using tag on its windscreen or license plate.

\subsection{Observations of Driver Survey}

1. Lot of time is wasted in finding a vacant parking spot.Instead of parking illegally or waiting for a vacant on street parking space, drivers prefer to go to the nearest parking area i.e. off street.

2. A considerable percentage of people prefer using smart phone for parking related information.

\subsection{Parking Information Systems}

a) Monitoring parking availability

1. Keeping track of vehicles at entry and exit points.

2. Ultrasonic sensors above each parking spot.

3. Magnetic sensors that senses change in magnetic field when vehicle is parked.

4. CCTV cameras and image processing techniques can be used to detect presence of vehicle.

b) Parking Guidance \& Information Systems

1. Mobile and Web applications to access real time data.

2. SPARK system uses vehicular communication via on board units and roadside units to monitor availability of parking slot and adds to security of vehicle.

3. The monitoring vehicle has GPS device and an ultrasonic sensor to check if a vehicle is already present on the slot.

\subsection{Smart Parking Concepts:}

a) Parking Reservation

1. Drivers must send a request and receive confirmation through SMS or internet.

2. Upon arrival, the driver and confirmation should be verified using RFID.

3. Once a booking is done, its validity needs to be considered.

b) Extra parking areas

Overstays can result in subsequent reservations not being honored. Some parking slots should be kept empty always to cover such cases.

c) Dynamic Parking

1. Charging more for parking in urban and industrial areas as compared to less crowded areas.

2. High pricing during peak hours will result in people opting for public transport which will reduce traffic.

\section{CROWDSOURCING AS A MODEL FOR PROBLEM SOLVING [4]}

Crowdsourcing is an online, distributed problem-solving and production model. Here, a company posts a problem online, a vast number of individuals offer solutions to the problem, the winning ideas are awarded some form of a bounty, and the company mass produces the idea for its own gain. Some successful examples of crowd-sourcing are Threadless, iStockPhoto and InnoCentive. The central idea is that the collective intelligence of the crowd is greater than that of a group of professionals and the web provides the necessary platform for crowd-sourcing. Crowd-sourcing differs from open-source development in the fact that, crowd-sourcing compensates the crowd for its efforts in problemsolving/product development and the solutions are owned by the company. The advantage is that it yields better solutions than closed-source model while overcoming drawbacks of open-source model and it offers the crowd an opportunity at entrepreneurship or at the least, a creative outlet. The downside is that the compensation for winning solutions is hardly comparable to the intellectual effort required to produce it and at times, it may ruin careers of professionals as the production is being done by the crowd without much expense.

\section{CROWD-ENHANCED CLOUD SERVICES: ISSUES AND DIRECTIONS [7]}

Cloud computing systems are distributed systems having interconnected computing resources (CPU, storage) are shared. It is a model for enabling convenient, on demand network access over the internet to shared pool of configurable computer resources with minimal management effort or service providers' interactions.

There are some cases in which cloud techniques solely are not able to do the task due to the nature of the task.

\subsection{Need for crowd-enhanced cloud systems}

There are many problems for which one or more computational services in combination with human agents can actually offer whole or part of a badly needed service that can achieve a level of service quality that cannot be achieved under each regime solely. Example: human evaluation of the output of a language translation job done by machine; human query processing to complement database query processing, big-data management approaches for large-scale classification of items.

Crowd-enhanced cloud services are distributed systems created as a result of injecting crowdsourcing into cloud computing at resource pool level (lowest level of cloud computing).

CloudVendors: Google,Amazon, Salesforce, Microsoft, Oracle Cloud.

IaaS: Virtualization of infrastructure resources. Example: Amazon EC2, Rackspace.

PaaS: Software Platform (OS, Software development framework, execution environment). Example: Google App Engine, MS Azure platform.

SaaS: Use of Applications over the internet, without local installation. Example: Amazon web services. 


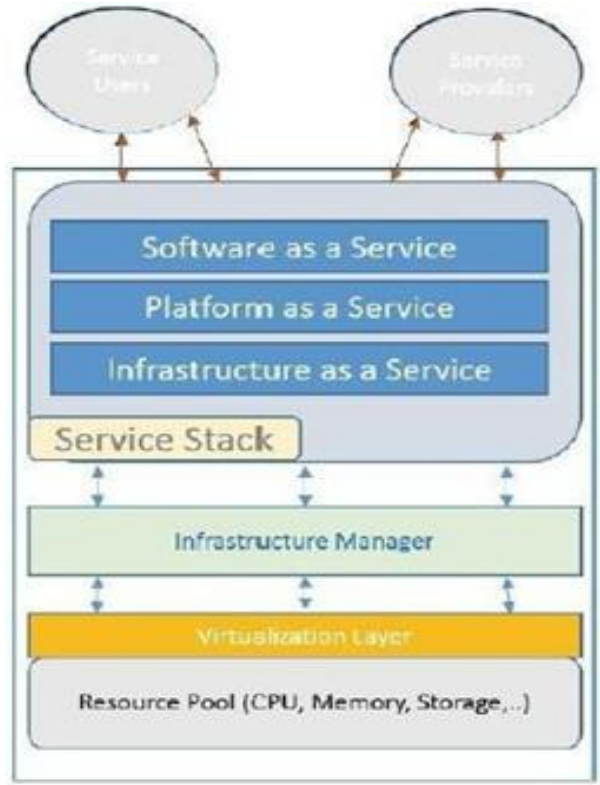

Figure 3 : Cloud Computing Diagram

\subsection{Crowdsourcing process}

The problem owner (requester) prepares a request (crowdsourcing task) for the crowd's contributions and submits it to a crowdsourcing platform.

People who are willing to contribute to the task (workers) select the task if they are eligible to do so, and provide the requester with their contributions through crowdsourcing platform or directly. The requester may evaluate the contributions and reward the workers.

\subsection{Crowdsourcing platforms}

AmazonMechanicalTurk1(MTurk), Crowdflower2,

Wikipedia3and Stackoverflow4

Injecting Cloud-

WHY? : Harnessing crowd intelligence and wisdom to solve a problem. People as computing resources (Human Intelligence Tasks).

WHERE? : At lowest level i.e. Resource pool.

\subsection{Drawbacks:}

No confidentiality, Poor quality entries, wrong direction

\section{CONCLUSION}

Due to advancement in technology, drivers are demanding easier and less time-consuming parking facilities. The various methodologies of smart parking that are described in this paper have enhanced the services provided to the end users and improved the overall management of the existing parking system. Many of the solutions proposed have already been successfully implemented while others are further being analyzed for implementation purpose. The real time monitoring of available parking lots and allotment of the suitable parking area by advanced reservation are some of the characteristic services provided to the customers or end users through a user friendly interface like a mobile application or using SMS services. After analyzing the different solutions, it is observed that there are some drawbacks of each system like excessive use of expensive sensor modules, difficulty in sensing accurate parking availability data due to speed constraints of the vehicle, use of certain modules like Bluetooth that function efficiently only for short distance, inefficient user authentication techniques and centralized management of the database of parking areas in different regions. These drawbacks need to be eliminated to enhance the overall performance of the system.

\section{REFERENCES}

[1] Z. Pala and N. Inanc, "Smart parkingapplications using RFID technology" in $1^{\text {st }}$ Annual Eurasia RFID conference, September 2007.

[2] Wand and W. He, "A reservation based smart parking system" in $1^{\text {st Int.'lWorkshop on Cyber-Physical }}$ networking systems, April 2011.

[3] N.H.H.M. Hanif, M.H. Badiozaman andH. Daud, "Smart parking reservation system using short message services (SMS)" in2010InternationalConference on Intelligent and Advanced Systems (ICIAS), June 2010.

[4] Brabham, "Crowdsourcing as a model for problem solving: An introduction and Cases" Convergence: TheInternational Journal of Research into New Media Technlogical Studies.

[5] S. Mathur, T. Jin, N. Kasturirangan, W. Xue, M. Gruteser and W. Trappe,"Parknet : drive by sensing of road-side parking statistics" in Proceedings of theEighth International Conference on Mobile Systems, applications and services, ACM New York, June 2010.

[6] Elena Polycarpou, LambrosLambrinos and EftychiosProtopapadakis, "SmartPaking Solutions for Urban Areas"

[7] Saeed Arbabi, Mohammad Allahbakhsh, Mohsen Sharifi, "Crowd-Enhanced Cloud Services: Issuesand Directions", International Journal of Computer Applications (0975 - 8887) Volume 117- No. 21, May 2015. 